Résumés des conférences et travaux

\title{
Histoire urbaine de l'Orient romain tardif
}

\section{Histoire urbaine de l'Orient romain tardif}

\section{Catherine Saliou}

\section{(2) OpenEdition \\ Journals}

Édition électronique

URL : https://journals.openedition.org/ashp/1943

DOI : $10.4000 /$ ashp. 1943

ISSN : 1969-6310

Éditeur

Publications de l'École Pratique des Hautes Études

\section{Édition imprimée}

Date de publication : 1 septembre 2017

Pagination : 122-127

ISSN : 0766-0677

\section{Référence électronique}

Catherine Saliou, "Histoire urbaine de l'Orient romain tardif », Annuaire de l'École pratique des hautes études (EPHE), Section des sciences historiques et philologiques [En ligne], 148|2017, mis en ligne le 25 septembre 2017, consulté le 06 juillet 2021. URL : http://journals.openedition.org/ashp/1943 ; DOI : https://doi.org/10.4000/ashp.1943 


\title{
HISTOIRE URBAINE DE L'ORIENT ROMAIN TARDIF
}

\author{
Directeur d'études : $\mathrm{M}^{\mathrm{me}}$ Catherine SAliou
}

Programme de l'année 2015-2016 : I. Construire dans l'Orient romain tardif : acteurs, contraintes, conflits. - II. Les sources écrites de l'histoire de l'espace urbain dans l'Orient romain tardif.

\section{Construire dans l'Orient romain tardif : acteurs, contraintes, conflits}

Le travail engagé les années précédentes à propos de la Loi de Zénon sur la construction privée à Constantinople (CJ VIII, 10, 12 ${ }^{1}$ ) a débouché sur des questions de portée plus générale, résumées dans l'intitulé « Construire dans l'Orient romain tardif : acteurs, contraintes, conflits ». On s'est intéressé en particulier cette année aux acteurs de la construction, et plus précisément aux « mécaniciens » ( $\mu \eta \chi \alpha v i \kappa o i ́)$ et aux « architectes » ( $\dot{\alpha} \rho \chi 1 \tau \varepsilon ́ \kappa \tau o v \varepsilon \varsigma)$, en prenant pour point de départ un article de Glanville Downey, "Byzantine architects: their training and methods ", Byzantion 18, 1946-1948, 99-118, qui a exercé une très forte influence sur la recherche postérieure. D’après G. Downey, les «mécaniciens » seraient dans l'Antiquité tardive les architectes par excellence, responsables de la conception des édifices, les professionnels désignés comme des « architectes » n'étant quant à eux que des chefs d'équipe. On a cherché à vérifier cette thèse.

On a commencé par dresser un tableau prosopographique des " mécaniciens » connus. Ce tableau a mis en évidence la diversité de leurs activités et de leurs compétences (astronomie, physique, géométrie, hydraulique, poliorcétique, fabrication de machines de guerre, arpentage, et enfin construction), ainsi que de leurs statuts et de leurs rangs. La prise en compte des mentions génériques de " mécaniciens » a permis de compléter et de préciser ces constatations. On a ensuite effectué le même travail pour les « architectes ». L'examen de l'ensemble de la documentation ainsi rassemblée a montré que les mots ả $\chi \imath \tau \varepsilon ́ \kappa \tau \omega v /$ architectus et $\mu \eta \chi \alpha v$ iós / mechanicus sont utilisés de façon apparemment indifférenciée pour désigner des spécialistes du génie militaire (machines de guerre, poliorcétique, remparts), mais aussi des spécialistes de l'hydraulique, ou encore de la mesure et de l'arpentage. Les mentions sûrement datées d'architectes concevant des édifices ou en dirigeant la construction sont rares après 512 . La première mention sans ambiguïté d'un mécanicien faisant œuvre d'architecte remonte au $\mathrm{II}^{\mathrm{e}}$ s. apr. J.-C. si l'Hippias de Lucien est bien authentique. Le témoignage de Grégoire de $\mathrm{Nysse}^{2}$ suggère que dès la fin $\mathrm{du}_{\mathrm{IV}}^{\mathrm{e}} \mathrm{s}$. il n'est pas rare que des mécaniciens soient impliqués dans des projets architecturaux, toutefois $\mathrm{c}^{\text {'est }} \mathrm{au} \mathrm{VI}^{\mathrm{e}} \mathrm{s}$. que se situe l'apogée de cette tendance, au moment même où les mentions d'architectes se font plus rares : un mécanicien construit ou au moins

1. Voir Annuaire 2013-2014, p. 109-110 et Annuaire 2014-2015, p. 114-117.

2. In sanctum Pascha (éd. E. Gebhardt, Gregorii Nysseni opera, 9, 1, Leyde, 1967), p. 257, 1. 23. 
conçoit l'église dite Néa à Jérusalem; à Constantinople, Sainte-Sophie est l'œuvre de deux mécaniciens, Isidore de Milet et Anthémios de Tralles; le mécanicien Chrysès d'Alexandrie est crédité de façon assez imprécise de divers travaux outre le barrage de Dara. L'activité des mécaniciens dans le domaine édilitaire ne se réduit pas toujours à la conception, ils peuvent aussi se charger de l'adjudication des travaux aux entrepreneurs et du suivi de la réalisation. Tous les mécaniciens par ailleurs ne font pas œuvre d'architecture, et il est possible que l'activité architecturale des mécaniciens corresponde dans la plupart des cas à une extension de leurs compétences en génie militaire et en hydraulique ou, dans le cas de la Néa à Jérusalem, à la spécificité du contexte d'implantation de l'édifice, imposant un aménagement du terrain requérant des compétences particulières. Ces remarques mettent en relief la singularité du cas de Sainte-Sophie. L'architecture et la mécanique sont par ailleurs des disciplines d'enseignement : le Tarif de Dioclétien mentionne l'architecture parmi les disciplines susceptibles de faire l'objet d'un enseignement privé, rémunéré de façon relativement faible; la législation impériale de la première moitié du $\mathrm{IV}^{\mathrm{e}} \mathrm{s}$. manifeste un souci d'encourager la formation des futurs architectes, mécaniciens et géomètres. Dans le cadre de cette législation, l'architecture relève des études supérieures, abordées à partir de 18 ans, après une formation en lettres. À la fin $\mathrm{du} \mathrm{IV}^{\mathrm{e}} \mathrm{s}$., le sénateur et comte Cyriades est qualifié de mechanicus (Symmaque, Rel. 25-26, ep. V, 6) et de mechanicae professor (Symmaque, Rel. 25). Le terme professor, toutefois, est ambigu et n'implique pas toujours une activité d'enseignement. $\mathrm{Au} \mathrm{VI}^{\mathrm{e}} \mathrm{s}$. Isidore de Milet a bien eu quant à lui une activité d'enseignement.

Dans l'Antiquité tardive, architecture et mécanique constituent donc deux disciplines distinctes qui se recoupent largement, mais pas totalement : l'activité scientifique est le fait des seuls mécaniciens, que l'on voit en particulier s'intéresser à l'astronomie, à la physique, à la géométrie, mais l'expertise juridique relève quant à elle des seuls architectes. Pour Vitruve, au $\mathrm{I}^{\text {er }}$ s. av. J.-C., la mécanique est une branche de l'architecture, et constitue donc un des domaines d'activité des architectes. Pour Galien également, au $\mathrm{II}^{\mathrm{e}}$ s., la mécanique fait partie de l'architecture. Pour comprendre l'évolution qui a abouti à l'autonomisation de la mécanique par rapport à l'architecture, il faut tenir compte de l'existence, au sein même des disciplines, de débats qui nous échappent en grande partie et qui s'inscrivent dans le cadre plus général d'une réflexion sur les relations entre la théorie et la pratique. On a relu le texte de Pappos d'Alexandrie ( $\mathrm{IV}^{\mathrm{e}} \mathrm{s}$.) qui joue un rôle clé dans la démonstration de G. Downey ${ }^{3}$. Notre lecture a été sensiblement différente de la sienne : Pappos mentionne, certes, la construction parmi les activités pratiques liées à la mécanique, mais dans un contexte où il peut s'agir précisément de la construction de machines, et il associe les architectes aux mécaniciens dans la mesure où ils ont besoin des mêmes notions de mathématiques et de géométrie, mais sans établir de hiérarchie entre eux. L'unique expression qui pourrait témoigner d'une supériorité de la mécanique sur l'architecture est à l'évidence une scolie postérieure. La présentation de Pappos met aussi en évidence la diversité des activités des mécaniciens et donc la polysémie du

3. Collection mathématique (éd. F. Hultsch, Pappi Alexandrini Collectionis quae supersunt, Berlin, 1876), VIII,1, p. 1022, 1. 15, p. 1024, 1. 2; VIII, 3, p. 1026, 1. 23, p. 1028, 1.1. 
terme $\mu \eta \chi \alpha v$ ıkós lui-même, qui peut s'appliquer à des personnes de niveau scientifique et de statut social variable. Cette lecture a été prolongée par celle d'un passage de $\operatorname{Proclos}^{4}\left(\mathrm{v}^{\mathrm{e}} \mathrm{s}\right.$.). Dans le cadre d'une présentation générale des mathématiques et de leurs usages, Proclos mentionne la mécanique parmi les mathématiques appliquées. Il reprend l'inventaire des différents domaines d'activités de la mécanique énumérés par Pappos, sans faire aucune référence ni à l'architecture ni à la construction, ce qui tend à confirmer que dans le texte de Pappos les mentions de ces disciplines ne doivent pas être surinterprétées et que l'objectif de Pappos n'est pas d'affirmer une hiérarchie entre architecture et mécanique. Asclépios de Tralles est finalement le premier auteur à signaler explicitement une hiérarchie entre le $\mu \eta \chi \alpha v ı \kappa o ́ s$, présenté comme un concepteur, et une figure qu'il désigne, non comme ả $\chi \imath \tau \varepsilon ́ \kappa \tau \omega v$, mais

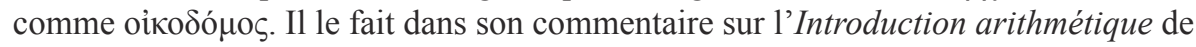
Nicomaque $^{5}$ (I, 19), qui doit être tiré de ses notes prises durant les cours d'Ammonios (ca 440-apr. 517). Simplicios ( ca 480-549) fournit un fondement théorique à cette hiérarchie dans son commentaire au De caelo d'Aristote ${ }^{6}$.

La construction d'une hiérarchie entre architecture et mécanique ne semble donc pas antérieure à la fin du $v^{\mathrm{e}} \mathrm{s}$. Cette hiérarchie théorique entre les disciplines ne doit pas être confondue avec une répartition hiérarchique des fonctions entre architectes et mécaniciens dans la réalisation des projets édilitaires, et chacun de ces deux groupes garde sa spécificité. On a rappelé pour conclure que dans la plupart des cas, d'après les inscriptions, la construction, dans l'Antiquité tardive, est le fait de professionnels qui ne se désignent ni comme des « mécaniciens » ni comme des « architectes »,

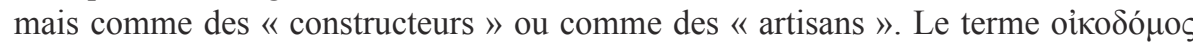
(« constructeur ») est fréquemment utilisé dans l'Antiquité tardive, dans divers contextes, et peut s'appliquer à des constructeurs dotés d'un haut niveau de qualification, voire alterner avec le terme ả $\chi \imath \tau \varepsilon ́ \kappa \tau \omega \nu$ à propos d'un même personnage. Le terme $\tau \varepsilon \chi v i ́ \tau\rceil \varsigma$ ( « artisan »), de signifié très général, peut prendre différents sens spécialisés, et peut notamment s'appliquer au maître d'œuvre d'une construction. Il faut également tenir compte du rôle joué par des non-professionnels, les commanditaires d'une part, la main d'œuvre non qualifiée d'autre part.

\section{Les sources écrites de l'histoire de l'espace urbain dans l'Orient romain tardif}

On a repris cette année l'étude des sources écrites de l'histoire du paysage urbain d'Antioche sur l'Oronte ${ }^{7}$. On a présenté le site d'Antioche et rappelé les principaux

4. In primum Euclidis elementorum librum (éd. G. Friedlein, Procli Diadochi in primum Euclidis elementorum librum commentarii, Leipzig 1873) p. 41, 1. 3 sqq.

5. Éd. L. Tarán, Asclepius of Tralles. Commentary to Nicomachus' introduction to arithmetic = Transactions of the American Philosophical Society, n.s. 59.4, Philadelphie, 1969.



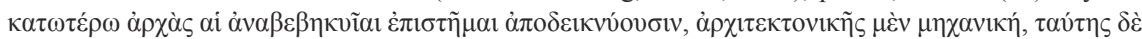

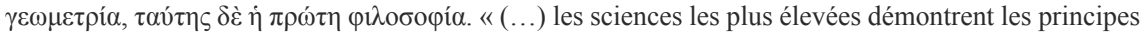
de celles qui leur sont inférieures : ceux de l'architecture, la mécanique, ceux de cette dernière, la géométrie, ceux de la géométrie, la philosophie première. »

7. Voir Annuaire 2013-2014, p. 107-109. 
traits de l'histoire de la ville. On a insisté en particulier sur les difficultés d'interprétation des textes relatifs aux espaces publics tels que l' « agora », le « forum de Valens », le « Macellum », difficultés encore soulignées par la découverte récente de vestiges susceptibles d'être identifiés à tel ou tel espace. Pour replacer dans leur contexte architectural et urbain les indications fournies par les sources écrites, et en particulier Jean Malalas, il faut substituer à une démarche narrative chronologique une démarche somme toute plus archéologique, partant des derniers états connaissables de la ville antique, en s'appuyant sur les témoignages les plus récents. On a commencé par celui de l'Histoire Ecclésiastique d'Évagre le Scolastique, rédigée entre 590 et $595^{8}$.

Après une présentation d'ensemble de l'ouvrage et l'analyse du récit du tremblement de terre de 588 , qui présente une dernière image d'ensemble de la ville à la fin $\mathrm{du} \mathrm{VI} \mathrm{e}^{\mathrm{e}} \mathrm{s}$, l'étude croisée des références à l'espace urbain d'Antioche et des renvois au présent dans l'ensemble du texte a montré que ces renvois étaient bien le fait d'Évagre lui-même et non d'une source antérieure, et a mis en évidence l'intérêt d'Évagre pour l'espace urbain antiochéen et la crédibilité de son témoignage. On a ensuite procédé à un inventaire analytique des mentions de lieux antiochéens dans l'Histoire Ecclésiastique, sous forme d'un tableau dont une première esquisse a été présentée et discutée, dans la perspective de l'élaboration d'une " carte mentale » de l'Antioche d'Évagre. Cet inventaire a mis en évidence quelques points. L'étude de la répartition dans l'ensemble de l'Histoire Ecclésiastique des désignations "Antioche », " cité d'Antiochos » « (cité) des Antiochéens » d'une part, et « Théoupolis » ou « cité des Théoupolites » d'autre part, a montré que « Théoupolis » n'est utilisé dans le récit d'Évagre qu'après la mention du changement de nom de la cité (IV, 6). La seule exception est l'occurrence de la formule « la (cité) des Théoupolites » au livre I, à propos du monastère d'Euprépios $(I, 7)$. Cette répartition suggère que dans ce dernier cas l'indication de localisation de ce monastère le situe bien par rapport aux limites de la ville de la fin du $\mathrm{VI}^{\mathrm{e}} \mathrm{s}$. L'étude de la répartition des emplois des mots $\beta \alpha \lambda \alpha v \varepsilon \tilde{o} \mathrm{v}$

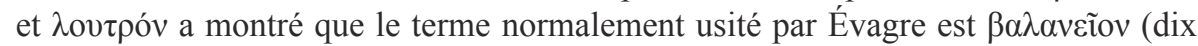
occurrences), alors que $\lambda$ ov $\tau$ óv n'apparaît que trois fois, d'une part en I, 19 à propos du financement de la restauration du bain de Valens par Eudocie, d'autre part, en deux

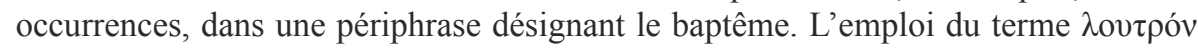
à propos du bain de Valens apparaît donc comme une anomalie, qui s'explique peutêtre par l'influence de la source utilisée par Évagre. Évagre ne mentionne que cinq églises et un monastère, et nomme en revanche de nombreux édifices civils : aménagements relevant de l'architecture connective (tétrapyle, portiques) ou des lieux de convergence (basiliques), édifices thermaux, " palais de justice », " résidences des stratèges », prison. L'identité de l'Antioche de l'Antiquité tardive ne saurait être réduite à la qualification de « ville chrétienne » et plus généralement ne saurait être définie par la religion.

On s'est intéressé plus précisément au chapitre I, 18, où sont énumérées diverses réalisations édilitaires dues à des représentants de l'État romain sous le règne de Théodose II. On s'est demandé à première lecture comment il fallait interpréter

8. Évagre le Scolastique, Histoire ecclésiastique, texte de l'édition Bidez-Parmentier, Londres, 1898, traduction A. J. Festugière, revue par L. Angliviel de la Baumelle et al., Paris, 2011-2014 (SC 542 et 566). 
l'alternance entre $\beta \alpha \sigma i ́ \lambda \varepsilon 10 \varsigma$ $\sigma \tau o \alpha ́$ et $\sigma \tau o \alpha ́$ au sein du chapitre. L'étude d'ensemble des occurrences de ces termes dans l'Histoire Ecclésiastique a permis de rappeler que le mot $\sigma \tau$ oó désigne souvent un portique intérieur, et a montré qu'il peut être utilisé comme le groupe $\beta \alpha \sigma i ́ \lambda \varepsilon$ lo $\sigma \tau o \alpha$ à propos d'édifices ou d'éléments d'édifices dési-


rale de ces termes, la prudence doit rester de règle.

Parmi les édifices mentionnés dans le chapitre I, 18, le " portique de Calliste »

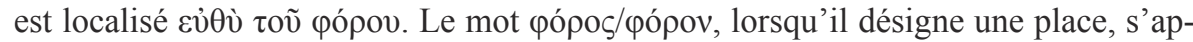
plique toujours à un forum impérial, susceptible de porter le nom d'un empereur. Le forum en question doit donc être le Forum de Valens. Le témoignage d'Évagre serait à cet égard d'autant plus précieux que Malalas est le seul autre auteur à parler de ce Forum. Mais il est possible, d'après le plus récent éditeur de la Chronique de Malalas, que ce passage d'Évagre soit tiré directement de la Chronique et permette de combler une lacune de la traduction manuscrite ${ }^{9}$. Si Évagre dépend de Malalas et ne constitue pas une source indépendante, l'intérêt de son témoignage est diminué. Il faut donc essayer d'évaluer plus précisément la relation entre le livre I de l'Histoire Ecclésiastique d'Évagre et les chapitres de Malalas consacrés au règne de Théodose II.

On a donc procédé à une étude comparée de la présentation du règne de Théodose II dans la Chronique de Malalas et dans l'Histoire Ecclésiastique d'Évagre. On s'est attaché d'abord à la comparaison de l'épisode du passage d'Eudocie à Antioche dans la Chronique telle qu'elle nous a été transmise sous forme résumée par le manuscrit $O$, la version non abrégée présente sous deux formes légèrement différentes dans le palimpseste de Tusculum et la Chronique Paschale, et l'Histoire Ecclésiastique d'Évagre. Les différences sont substantielles : mention de deux statues ou d'une seule, localisation ou non du discours d'Eudocie et des statues, et surtout destination différente du don en numéraire. Aucun élément ne permet d'établir la dépendance d'Évagre par rapport à Malalas pour ce passage. Plus généralement, les différences entre Malalas et Évagre, pour le récit du règne de Théodose II, sont nombreuses. Elles s'expliquent par les différences d'objectifs des deux auteurs, et plus précisément par l'insertion dans le récit de Malalas du « roman d'Eudocie » incluant l'épisode du don de la pomme à Paulin, alors qu'Évagre omet cette histoire scandaleuse. De plus Malalas attribue l'extension du rempart de la ville à Théodose $\mathrm{I}^{\mathrm{er}}$ et non Théodose II. Le fait qu' Évagre relève cette erreur — sans signaler explicitement qu'elle est de Malalas - signale la connaissance qu'il a de la Chronique mais n'implique pas qu'il l'ait utilisée comme source. Toutefois, dans le cas précis du chapitre I, 18, l'utilisation de Malalas n'est pas à exclure, et un argument serait la présence dans ce passage de mentions d'édifices à fonction exclusivement topologique (pour situer d'autres édifices), typiques de Malalas, mais absentes par ailleurs de l'œuvre d’Évagre quand il s'agit d'Antioche. La prudence doit cependant rester de mise et faire de l'Histoire Ecclésiastique une source permettant de reconstruire le texte de Malalas est en tout état de cause excessif.

9. H. Thurn, Ioannis Malalae chronographia, Berlin, New York, 2000 (CFHB 35), XIV, 13, p. 280, apparat critique. 
Après ce détour, on est revenu à l'étude de détail de la phrase mentionnant le

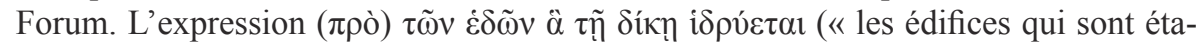
blis pour la justice ») peut être rapprochée de formules épigrammatiques désignant comme « le sanctuaire de la Justice », voire tout simplement « la Justice », le prétoire d'un gouverneur, ou à Constantinople celui du Préfet de la Ville ${ }^{10}$. Dans la mesure où il se trouve bien au " Forum de Valens ", l'édifice ainsi désigné est le prétoire du consulaire de Syrie : le passage d'Évagre fournit ainsi un repère chronologique pour la transformation du bain de Commode en prétoire du gouverneur de Syrie, signalée par Malalas comme postérieure à l'aménagement du Forum par Valens. Elle devait être effective vers 430 au plus tard. Un exemple d'une transformation partielle d'un établissement thermal en salle d'audience ou « prétoire » est fourni par le « prétoire de Gortyne » en Crète, qui fait aussi partie des exemples de prétoire désignés comme «la Justice» dans plusieurs inscriptions ${ }^{11}$.

Deux séances ont été assurées respectivement par Richard Bouchon, maître de conférences à l'université Lyon II (« Le théâtre de Larissa [Thessalie] : épigraphie des gradins d'édifices de spectacles dans le monde grec d'époque romaine $»)$, le 12 mai 2016 et par Giovanni Alberto Cecconi, professeur à l’université de Florence (« Éléments de conflit religieux dans les écoles publiques de l'empire tardif : lecture de sources connues et moins connues »), le 26 mai 2016.

10. Ex. : IG VII, 94-95; Anth. IX, 658-659, 812- 813.

11. I. Cret. IV 313, 323, 325; G. Vallarino, « Epigramma dedicatorio per uno hyparchos dall'area del Pretorio di Gortina », Zeitschrift für Papyrologie und Epigraphik 183, 2012, p. 59-66. 